\title{
Related Fixed Point Theorems for Three Metric Spaces, II
}

\author{
Sampada Navshinde, Dr. J. Achari and Brian Fisher
}

Abstract. In this paper, we have proved some related fixed point theorems for three metric spaces which improve the results of Jain, Sahu and Fisher [2].

\section{INTRODUCTION}

The following fixed point theorem was proved by Jain, Sahu and Fisher [2].

Theorem 1. Let $(X, d),(Y, \rho)$ and $(Z, \sigma)$ be complete metric spaces. If $T$ is continuous mapping of $X$ into $Y, S$ is a mapping of $Y$ into $Z$ and $R$ is a mapping of $Z$ into $X$ satisfying the inequalities

$$
\begin{gathered}
d\left(R S T x, R S T x^{\prime}\right) \leq c \max \left\{d\left(x, x^{\prime}\right), d(x, R S T x), d\left(x^{\prime}, R S T x^{\prime}\right),\right. \\
\left.\quad \rho\left(T x, T x^{\prime}\right), \sigma\left(S T x, S T x^{\prime}\right)\right\}, \\
\rho\left(T R S y, T R S y^{\prime}\right) \leq c \max \left\{\rho\left(y, y^{\prime}\right), \rho(y, T R S y), \rho\left(y^{\prime}, T R S y^{\prime}\right),\right. \\
\left.\quad \sigma\left(s y, s y^{\prime}\right), d\left(R S y, R S y^{\prime}\right)\right\}, \\
\sigma\left(S T R z, S T R z^{\prime}\right) \leq c \max \left\{\sigma\left(z, z^{\prime}\right), \sigma(z, S T R z), \sigma\left(z^{\prime}, S T R z^{\prime}\right)\right. \\
\left.d\left(R z, R z^{\prime}\right), \rho\left(T R z, T R z^{\prime}\right)\right\}
\end{gathered}
$$

for all $x, x^{\prime}$ in $X, y, y^{\prime}$ in $Y$ and $z, z^{\prime}$ in $Z$, where $0 \leq c<1$, then RST has a unique fixed point $u$ in $X, T R S$ has a unique fixed point $v$ in $Y$ and $S T R$ has a unique fixed point $w$ in $Z$. Further, $T u=v, S v=v$, and $R w=u$.

\section{Main Results}

We now prove the following related fixed point theorem which improves Theorem 1.

2000 Mathematics Subject Classification. Primary: 54H25.

Key words and phrases. Complete metric space, compact metric space, related fixed point. 
Theorem 2. Let $(X, d),(Y, \rho)$ and $(Z, \sigma)$ be complete metric spaces. If $T$ is continuous mapping of $X$ into $Y, S$ is a mapping of $Y$ into $Z$ and $R$ is a mapping of $Z$ into $X$ satisfying the inequalities

(1)

$$
\begin{aligned}
d\left(R S T x, R S T x^{\prime}\right) \leq c & \max \left\{\frac{d\left(x, x^{\prime}\right)[1+d(x, R S T x)]}{1+d\left(x, x^{\prime}\right)},\right. \\
& \frac{d\left(x^{\prime}, R S T x\right)\left[1+d\left(x, R S T x^{\prime}\right)\right]}{1+d\left(x, x^{\prime}\right)}, \\
& \frac{d\left(x^{\prime}, R S T x^{\prime}\right)[1+d(x, R S T x)]}{1+d\left(x, x^{\prime}\right)}, \\
& \left.\rho\left(T x, T x^{\prime}\right), \sigma\left(S T x, S T x^{\prime}\right)\right\}, \\
\rho\left(T R S y, T R S y^{\prime}\right) \leq c & \max \left\{\frac{\rho\left(y, y^{\prime}\right)[1+\rho(y, T R S y)]}{1+\rho\left(y, y^{\prime}\right)},\right. \\
& \frac{\rho\left(y^{\prime}, T R S y\right)\left[1+\rho\left(y, T R S y^{\prime}\right)\right]}{1+\rho\left(y, y^{\prime}\right)}, \\
& \frac{\rho\left(y^{\prime}, T R S y^{\prime}\right)[1+\rho(y, T R S y)]}{1+\rho\left(y, y^{\prime}\right)}, \\
& \left.\sigma\left(S y, S y^{\prime}\right), d\left(R S y, R S y^{\prime}\right)\right\}, \\
\sigma\left(S T R z, S T R z^{\prime}\right) \leq c & \max \left\{\frac{\sigma\left(z, z^{\prime}\right)[1+\sigma(z, S T R z)]}{1+\sigma\left(z, z^{\prime}\right)},\right. \\
& \frac{\sigma\left(z^{\prime}, S T R z\right)\left[1+\sigma\left(z, S T R z^{\prime}\right)\right]}{1+\sigma\left(z, z^{\prime}\right)}, \\
& \frac{\sigma\left(z^{\prime}, S T R z^{\prime}\right)[1+\sigma(z, S T R z)]}{1+\sigma\left(z, z^{\prime}\right)}, \\
& \left.d\left(R z, R z^{\prime}\right), \rho\left(T R z, T R z^{\prime}\right)\right\}
\end{aligned}
$$

for all $x, x^{\prime}$ in $X, y, y^{\prime}$ in $Y$ and $z, z^{\prime}$ in $Z$, where $0 \leq c<1$, then $R S T$ has a unique fixed point $u$ in $X, T R S$ has a unique fixed point $v$ in $Y$ and $S T R$ has a unique fixed point $w$ in $Z$. Further, $T u=v, S v=w$ and $R w=u$.

Proof. Let $x_{0}$ be an arbitrary point in $X$. Define sequences $\left\{x_{n}\right\},\left\{y_{n}\right\}$ and $\left\{z_{n}\right\}$ in $X, Y$ and $Z$ respectively by

$$
x_{n}=(R S T)^{n} x_{0}, \quad y_{n}=T x_{n-1}, \quad z_{n}=S y_{n}
$$

for $n=1,2, \ldots$ 
Applying inequality (2), we have

(4)

$$
\begin{aligned}
\rho\left(y_{n}, y_{n+1}\right)= & \rho\left(T R S y_{n-1}, T R S y_{n}\right) \\
\leq c \max \left\{\frac{\rho\left(y_{n-1}, y_{n}\right)\left[1+\rho\left(y_{n-1}, T R S y_{n-1}\right)\right]}{1+\rho\left(y_{n-1}, y_{n}\right)},\right. & \frac{\rho\left(y_{n}, T R S y_{n-1}\right)\left[1+\rho\left(y_{n-1}, T R S y_{n}\right)\right]}{1+\rho\left(y_{n-1}, y_{n}\right)}, \\
& \frac{\rho\left(y_{n}, T R S y_{n}\right)\left[1+\rho\left(y_{n-1}, T R S y_{n-1}\right)\right]}{1+\rho\left(y_{n-1}, y_{n}\right)}, \\
& \left.\sigma\left(S y_{n-1}, S y_{n}\right), d\left(R S y_{n-1}, R S y_{n}\right)\right\}, \\
\leq & \max \left\{\rho\left(y_{n-1}, y_{n}\right), \sigma\left(z_{n-1}, z_{n}\right), d\left(x_{n-1}, x_{n}\right)\right\} .
\end{aligned}
$$

Using inequality (3), we have

$$
\begin{aligned}
& \sigma\left(z_{n}, z_{n+1}\right)= \sigma\left(S T R z_{n-1}, S T R z_{n}\right) \\
& \leq c \max \left\{\frac{\sigma\left(z_{n-1}, z_{n}\right)\left[1+\sigma\left(z_{n-1}, S T R z_{n-1}\right)\right]}{1+\sigma\left(z_{n-1}, z_{n}\right)}\right. \frac{\sigma\left(z_{n}, S T R z_{n-1}\right)\left[1+\sigma\left(z_{n-1}, S T R z_{n}\right)\right]}{1+\sigma\left(z_{n-1}, z_{n}\right)}, \\
& \frac{\sigma\left(z_{n}, S T R z_{n}\right)\left[1+\sigma\left(z_{n-1}, S T R z_{n-1}\right)\right]}{1+\sigma\left(z_{n-1}, z_{n}\right)}, \\
&\left.d\left(R z_{n-1}, R z_{n}\right), \rho\left(T R z_{n-1}, T R z_{n}\right)\right\} \\
& \leq c \max \left\{\rho\left(y_{n-1}, y_{n}\right), \sigma\left(z_{n-1}, z_{n}\right), d\left(x_{n-1}, x_{n}\right)\right\}
\end{aligned}
$$

on using inequality (4).

Using inequality (1) we have

$$
\begin{aligned}
& d\left(x_{n}, x_{n+1}\right)=d\left(R S T x_{n-1}, R S T x_{n}\right) \\
& \quad \leq c \max \left\{\rho\left(y_{n+1}, y_{n}\right), \sigma\left(z_{n+1}, z_{n}\right), d\left(x_{n+1}, x_{n}\right), d\left(x_{n-1}, x_{n}\right)\right\} \\
& \quad \leq c \max \left\{\rho\left(y_{n-1}, y_{n}\right), \sigma\left(z_{n-1}, z_{n}\right), d\left(x_{n-1}, x_{n}\right)\right\},
\end{aligned}
$$

on using inequalities (4) and (5).

It follows easily by induction on using inequalities (4),(5) and (6) that

$$
\begin{gathered}
d\left(x_{n}, x_{n+1}\right) \leq c^{n-1} \max \left\{d\left(x_{1}, x_{2}\right), \rho\left(y_{1}, y_{2}\right), \sigma\left(z_{1}, z_{2}\right)\right\}, \\
\rho\left(y_{n}, y_{n+1}\right) \leq c^{n-1} \max \left\{d\left(x_{1}, x_{2}\right), \rho\left(y_{1}, y_{2}\right), \sigma\left(z_{1}, z_{2}\right)\right\}, \\
\sigma\left(z_{n}, z_{n+1}\right) \leq c^{n-1} \max \left\{d\left(x_{1}, x_{2}\right), \rho\left(y_{1}, y_{2}\right), \sigma\left(z_{1}, z_{2}\right)\right\} .
\end{gathered}
$$

Since $c<1$, it follows that $\left\{x_{n}\right\},\left\{y_{n}\right\}$ and $\left\{z_{n}\right\}$ are Cauchy sequences with limits $u, v$ and $w$ in $X, Y$ and $Z$ respectively. 
Since $T$ and $S$ are continuous, we have

$$
\begin{aligned}
& \lim _{n \rightarrow \infty} y_{n}=\lim _{n \rightarrow \infty} T x_{n}=T u=v, \\
& \lim _{n \rightarrow \infty} z_{n}=\lim _{n \rightarrow \infty} S y_{n}=S v=w .
\end{aligned}
$$

Using inequality (1) again, we have

$$
\begin{aligned}
d( & \left.R S T u, x_{n}\right)=d\left(R S T u, R S T x_{n-1}\right) \\
\leq & c \max \left\{\frac{d\left(u, x_{n-1}\right)[1+d(u, R S T u)]}{1+d\left(u, x_{n-1}\right)}, \frac{d\left(x_{n-1}, R S T u\right)\left[1+d\left(u, R S T x_{n-1}\right)\right]}{1+d\left(u, x_{n-1}\right)},\right. \\
& \left.\frac{d\left(x_{n-1}, R S T x_{n-1}\right)[1+d(u, R S T u)]}{1+d\left(u, x_{n-1}\right)}, \rho\left(T u, T x_{n-1}\right), \sigma\left(S T u, S T x_{n-1}\right)\right\}, \\
\leq & c \max \left\{\frac{d\left(u, x_{n-1}\right)[1+d(u, R S T u)]}{1+d\left(u, x_{n-1}\right)}, \frac{d\left(x_{n-1}, R S T u\right)\left[1+d\left(u, x_{n}\right)\right]}{1+d\left(u, x_{n-1}\right)},\right. \\
& \left.\frac{d\left(x_{n-1}, x_{n}\right)[1+d(u, R S T u)]}{1+d\left(u, x_{n-1}\right)}, \rho\left(T u, T x_{n-1}\right), \sigma\left(S T u, S T x_{n-1}\right)\right\} .
\end{aligned}
$$

Since $S$ and $T$ are continuous, it follows on letting $n \rightarrow \infty$ that

$$
d(R S T u, u) \leq c d(R S T u, u) .
$$

Thus $R S T u=u$, since $c<1$ and so $u$ is a fixed point of $R S T$.

We therefore have

$$
T R S v=T R S T u=T u=v
$$

and so

$$
S T R w=S T R S v=S v=w .
$$

Hence $v$ and $w$ are fixed points of $T R S$ and $S T R$ respectively.

We now prove the uniqueness of the fixed point $u$. Suppose that $R S T$ has a second fixed point $u^{\prime}$. Then using inequality (1), we have

$$
\begin{aligned}
d\left(u, u^{\prime}\right)= & d\left(R S T u, R S T u^{\prime}\right) \\
\leq & c \max \left\{\frac{d\left(u, u^{\prime}\right)[1+d(u, R S T u)]}{1+d\left(u, u^{\prime}\right)}, \frac{d\left(u^{\prime}, R S T u\right)\left[1+d\left(u, R S T u^{\prime}\right)\right]}{1+d\left(u, u^{\prime}\right)},\right. \\
& \left.\frac{d\left(u^{\prime}, R S T u^{\prime}\right)[1+d(u, R S T u)]}{1+d\left(u, u^{\prime}\right)}, \rho\left(T u, T u^{\prime}\right), \sigma\left(S T u, S T u^{\prime}\right)\right\}, \\
\leq & c \max \left\{\frac{d\left(u, u^{\prime}\right)[1+d(u, u)]}{1+d\left(u, u^{\prime}\right)}, \frac{d\left(u^{\prime}, u\right)\left[1+d\left(u, u^{\prime}\right)\right]}{1+d\left(u, u^{\prime}\right)},\right. \\
& \left.\frac{d\left(u^{\prime}, u^{\prime}\right)[1+d(u, u)]}{1+d\left(u, u^{\prime}\right)}, \rho\left(T u, T u^{\prime}\right), \sigma\left(S T u, S T u^{\prime}\right)\right\}, \\
= & c \max \left\{\rho\left(T u, T u^{\prime}\right), \sigma\left(S T u, S T u^{\prime}\right)\right\} .
\end{aligned}
$$


Further, using inequality (2), we have

$$
\begin{aligned}
\rho\left(T u, T u^{\prime}\right)= & \rho\left(T R S T u, T R S T u^{\prime}\right) \\
\leq & c \max \left\{\frac{\rho\left(T u, T u^{\prime}\right)[1+\rho(T u, T R S T u)]}{1+\rho\left(T u, T u^{\prime}\right)},\right. \\
& \frac{\rho\left(T u^{\prime}, T R S T u\right)\left[1+\rho\left(T u, T R S T u^{\prime}\right)\right]}{1+\rho\left(T u, T u^{\prime}\right)}, \\
& \frac{\rho\left(T u^{\prime}, T R S T u^{\prime}\right)[1+\rho(T u, T R S T u)]}{1+\rho\left(T u, T u^{\prime}\right)}, \\
& \left.d\left(R S T u, R S T u^{\prime}\right), \sigma\left(S T u, S T u^{\prime}\right)\right\}, \\
\leq & c \max \left\{\frac{\rho\left(T u, T u^{\prime}\right)[1+\rho(T u, T u)]}{1+\rho\left(T u, T u^{\prime}\right)}, \frac{\rho\left(T u^{\prime}, T u\right)\left[1+\rho\left(T u, T u^{\prime}\right)\right]}{1+\rho\left(T u, T u^{\prime}\right)},\right. \\
& \left.\frac{\rho\left(T u^{\prime}, T u^{\prime}\right)[1+\rho(T u, T u)]}{1+\rho\left(T u, T u^{\prime}\right)}, d\left(u, u^{\prime}\right), \sigma\left(S T u, S T u^{\prime}\right)\right\}, \\
= & c \max \left\{d\left(u, u^{\prime}\right), \sigma\left(S T u, S T u^{\prime}\right)\right\} .
\end{aligned}
$$

Hence we have

$$
d\left(u, u^{\prime}\right) \leq c \sigma\left(S T u, S T u^{\prime}\right) .
$$

Finally, on using inequality (3), we have

$$
\begin{aligned}
d\left(u, u^{\prime}\right) \leq & c \sigma\left(S T u, S T u^{\prime}\right) \\
\leq & c \sigma\left(S T R S T u, S T R S T u^{\prime}\right) \\
\leq & c^{2} \max \left\{\frac{\sigma\left(S T u, S T u^{\prime}\right)[1+\sigma(S T u, S T R S T u)]}{1+\sigma\left(S T u, S T u^{\prime}\right)},\right. \\
& \frac{\sigma\left(S T u^{\prime}, S T R S T u\right)\left[1+\sigma\left(S T u, S T R S T u^{\prime}\right)\right]}{1+\sigma\left(S T u, S T u^{\prime}\right)}, \\
& \frac{\sigma\left(S T u^{\prime}, S T R S T u^{\prime}\right)[1+\sigma(S T u, S T R S T u)]}{1+\sigma\left(S T u, S T u^{\prime}\right)}, \\
& \left.d\left(R S T u, R S T u^{\prime}\right), \rho\left(T R S T u, T R S T u^{\prime}\right)\right\} \\
= & c^{2} d\left(u, u^{\prime}\right) .
\end{aligned}
$$

Since $c<1$, it follows that $u=u^{\prime}$ and the uniqueness of $u$ follows.

Similarly, it can be proved that $v$ is the unique fixed point of $T R S$ and $w$ is the unique fixed point of $S T R$.

We finally prove that we also have $R w=u$. To do this, note that

$$
R w=R(S T R w)=R S T(R w)
$$

and so $R w$ is a fixed point of $R S T$. Since $u$ is the unique fixed point of $R S T$, it follows that $R w=u$. This completes the proof of the theorem.

We now prove an analogous result for compact metric spaces. 
Theorem 3. Let $(X, d),(Y, \rho)$ and $(Z, \sigma)$ be compact metric spaces. If $T$ is continuous mapping of $X$ into $Y, S$ is a continuous mapping of $Y$ into $Z$ and $R$ is a continuous mapping of $Z$ into $X$ satisfying the inequalities

$$
\begin{aligned}
& d\left(R S T x, R S T x^{\prime}\right)< \\
&<c \max \left\{\frac{d\left(x, x^{\prime}\right)[1+d(x, R S T x)]}{1+d\left(x, x^{\prime}\right)}, \frac{d\left(x^{\prime}, R S T x\right)\left[1+d\left(x, R S T x^{\prime}\right)\right]}{1+d\left(x, x^{\prime}\right)},\right.\left.\frac{d\left(x^{\prime}, R S T x^{\prime}\right)[1+d(x, R S T x)]}{1+d\left(x, x^{\prime}\right)}, \rho\left(T x, T x^{\prime}\right), \sigma\left(S T x, S T x^{\prime}\right)\right\}, \\
& \rho\left(T R S y, T R S y^{\prime}\right)< \\
&<c \max \left\{\frac{\rho\left(y, y^{\prime}\right)[1+\rho(y, T R S y)]}{1+\rho\left(y, y^{\prime}\right)}, \frac{\rho\left(y^{\prime}, T R S y\right)\left[1+\rho\left(y, T R S y^{\prime}\right)\right]}{1+\rho\left(y, y^{\prime}\right)},\right. \\
&\left.\quad \frac{\rho\left(y^{\prime}, T R S y^{\prime}\right)[1+\rho(y, T R S y)]}{1+\rho\left(y, y^{\prime}\right)}, \sigma\left(S y, S y^{\prime}\right), d\left(R S y, R S y^{\prime}\right)\right\}, \\
& \sigma\left(S T R z, S T R z^{\prime}\right)< \\
&<c \max \left\{\frac{\sigma\left(z, z^{\prime}\right)[1+\sigma(z, S T R z)]}{1+\sigma\left(z, z^{\prime}\right)}, \frac{\sigma\left(z^{\prime}, S T R z\right)\left[1+\sigma\left(z, S T R z^{\prime}\right)\right]}{1+\sigma\left(z, z^{\prime}\right)},\right. \\
&\left.\quad \frac{\sigma\left(z^{\prime}, S T R z^{\prime}\right)[1+\sigma(z, S T R z)]}{1+\sigma\left(z, z^{\prime}\right)}, d\left(R z, R z^{\prime}\right), \rho\left(T R z, T R z^{\prime}\right)\right\}
\end{aligned}
$$

for all distinct $x, x^{\prime}$ in $X$, all distinct $y, y^{\prime}$ in $Y$ and all distinct $z, z^{\prime}$ in $Z$. Then RST has a unique fixed point $u$ in $X, T R S$ has a unique fixed point $v$ in $Y$ and $S T R$ has a unique fixed point $w$ in $Z$. Further, $T u=v, S v=w$ and $R w=u$.

Proof. Let us denote the right-hand side of inequalities (7), (8) and (9) by $h\left(x, x^{\prime}\right), k\left(y, y^{\prime}\right)$ and $p\left(z, z^{\prime}\right)$ respectively.

Suppose first of all that there exist $u, u^{\prime}$ in $X$ such that $h\left(u, u^{\prime}\right)=0$. Then it follows immediately that $u=u^{\prime}$ and $R S T u=u$. Then on putting $T u=v$, $S v=w$, we have

$$
\begin{aligned}
R S v=u \quad & \Rightarrow \quad T R S v=T u=v \\
S T R S v=S T R w=S v=w \quad & \Rightarrow \quad R S v=R w=u .
\end{aligned}
$$

The result of the theorem therefore holds in this case.

Similarly, if there exist $v, v^{\prime}$ in Y such that $k\left(v, v^{\prime}\right)=0$ or if there exist $w, w^{\prime}$ in $\mathrm{Z}$ such that $p\left(w, w^{\prime}\right)=0$, then the results of the theorem also hold.

Now suppose that $h\left(x, x^{\prime}\right) \neq 0$ for all $x, x^{\prime}$ in $X, k\left(y, y^{\prime}\right) \neq 0$ for all $y, y^{\prime}$ in $Y$ and $p\left(z, z^{\prime}\right) \neq 0$ for all $z, z^{\prime}$ in $Z$. Define the function $f$ on $X^{2}$ by

$$
f\left(x, x^{\prime}\right)=\frac{d\left(R S T x, R S T x^{\prime}\right)}{h\left(x, x^{\prime}\right)} .
$$


Then $f$ is continuous and since $X \times X$ is compact, $f$ attains its maximum value $c_{1}$. Because of inequality (7), $c_{1}<1$ and so

$$
d\left(R S T x, R S T x^{\prime}\right) \leq c_{1} h\left(x, x^{\prime}\right),
$$

for all $x, x^{\prime}$ in $X$.

Similarly, there exist $c_{2}, c_{3}<1$ such that

$$
\rho\left(T R S y, T R S y^{\prime}\right) \leq c_{2} k\left(y, y^{\prime}\right),
$$

for all $y, y^{\prime}$ in $Y$ and

$$
\sigma\left(S T R z, S T R z^{\prime}\right) \leq c_{3} p\left(z, z^{\prime}\right)
$$

for all $z, z^{\prime}$ in $Z$. It follows that the conditions of Theorem 2 are satisfied with $c=\max \left(c_{1}, c_{2}, c_{3}\right)$ and so the results of the theorem are again satisfied.

The uniqueness of $u, v$, and $w$ follows easily.

\section{REFERENCES}

[1] B. Fisher, Related fixed point theorem on two metric spaces, Math. Sem. Notes, Kobe Univ., 10 (1982), 17-26.

[2] R.K. Jain, H.K. Sahu and B. Fisher, Related fixed point theorem for three metric spaces, Novi Sad J. Math., 26(1) (1996), 11-17.

SAMPADA NAVSHINDE

H.No. 104, "Atrey", Konark Vihar

FARANDE NAGAR, WADI(BK)

NANDED-431605 (MAHARASHTRA)

INDIA

E-mail address: snavshinde@gmail.com

\section{Dr. J. Achari}

RAMANAND NAGAR, PAWDEWAdi NAKA NANDED (Maharashtra)

INDIA

\section{Brian Fisher}

Department of Mathematics

UNIVERSITY OF LEICESTER

LEICESTER, LE1 7RH

U.K.

E-mail address: fbr@le.ac.uk 
